\title{
Avaliação de extratos aquosos de várias espécies vegetais, aplicados ao solo, sobre Meloidogyne javanica (Treub, 1885) Chitwood, 1949
}

\author{
Evaluation of plant aqueous extracts, added into the soil, on \\ Meloidogyne javanica (Treub, 1885) Chitwood, 1949
}

\author{
Cristiane Gonçalves Gardiano ${ }^{1 *}$; Silamar Ferraz²; Everaldo Antônio Lopes ${ }^{3}$; \\ Paulo Afonso Ferreira ${ }^{4}$; Deisy Xavier Amora ${ }^{4}$; Leandro Grassi de Freitas ${ }^{2}$
}

\section{Resumo}

O uso de extratos vegetais com propriedades nematicidas no controle de fitonematóides representa mais uma alternativa para os pequenos produtores, com valor prático e econômico, e sem riscos de contaminação do ambiente. A adição ao solo dos extratos aquosos de 20 espécies de plantas foi avaliada sobre a população de Meloidogyne javanica em plantas de tomateiro, em casa de vegetação. Estas foram divididas em dois grupos e avaliadas em dois experimentos separados. No mesmo dia em que se infestou o solo com 5.000 ovos do nematóide, adicionou-se $20 \mathrm{~mL}$ dos extratos aquosos obtidos de folhas de artemísia (Chrysanthemum parthenium), bardana (Arctium lappa), capim cidreira (Cymbopogon citratus), carqueja (Bacharis trimera), cavalinha (Equisetum sp.), cinamomo (Melia azedarach), hortelã (Mentha sp.), mamona (Ricinus communis), manjericão (Ocimum basilicum), melão-de-SãoCaetano (Momordica charantia), arruda (Ruta graveolens), falso-boldo (Coleus barbatus), confrei (Symphitum officinalis), erva-de-bicho (Polygonum acre), feijão-de-porco (Canavalia ensiformis), funcho (Foeniculum vulgare), guiné (Petiveria alliacea), mentrasto (Ageratum conyzoides), mucunacinza (Mucuna pruriens) e nim (Azadirachta indica). Água destilada foi adicionada ao solo como tratamento testemunha. Após 60 dias, avaliou-se a altura das plantas, o peso fresco da parte aérea e da raiz e os números de galhas e de ovos por sistema radicular. Os extratos de hortelã, bardana e mamona reduziram o número de galhas em $75,6 \%, 65,7 \%$ e $54,4 \%$, e o número de ovos em $81,7 \%, 75,9 \%$ e $56,6 \%$, respectivamente.

Palavras-chave: Fitonematóides, controle alternativo, nematóides das galhas, compostos naturais

\begin{abstract}
The use of plant extracts with nematicidal properties to control plant nematodes can be a useful alternative to small farmers, regarding its low cost and non-toxic effects. The effect of the addition into the soil of aqueous extracts of 20 plant species on Meloidogyne javanica in plants of tomato was studied at greenhouse conditions. These were divided into two groups and evaluated in two separate experiments. The soil in the $2.0 \mathrm{~L}$ pots was infested with 5,000 eggs and, in the same day, it was applied $20 \mathrm{~mL}$ of aqueous extracts of the 20 plant species (Chrysanthemum parthenium, Arctium lappa, Cymbopogon
\end{abstract}

\footnotetext{
1 Enga Agrônoma, Parte da Dissertação de Mestrado em Fitopatologia na Universidade Federal de Viçosa - Universidade Estadual de Londrina, Departamento de Agronomia - Laboratório de Fitopatologia - C. P 6001, CEP 86051-990, Londrina, PR, Brasil. E-mail: cris gardiano@yahoo.com.br

2 Professor Doutor PhD no Depto de Fitopatologia, Universidade Federal de Viçosa, Departamento de Fitopatologia.

3 Eng ${ }^{\circ}$ Agrônomo, Aluno de Doutorado em Fitopatologia na Universidade Federal de Viçosa, Departamento de Fitopatologia.

4 Alunos de graduação em Agronomia na Universidade Federal de Viçosa, Departamento de Fitopatologia

* Autor para correspondência
} 
citratus, Bacharis trimera, Equisetum sp., Melia azedarach, Mentha sp., Ricinus communis, Ocimum basilicum, Momordica charantia, Ruta graveolens, Coleus barbatus, Symphitum officinalis, Polygonum acre, Canavalia ensiformis, Foeniculum vulgare, Petiveria alliacea, Ageratum conyzoides, Mucuna pruriens and Azadirachta indica). Distilled water was used as control. Sixty days later, were evaluated for plant height, the fresh weight of shoot and root and the numbers of galls and eggs per root system. The extracts of Mentha sp., Arctium lappa and Ricinus communis reduced the number of galls of M. javanica in $75.6 \% ; 65.7 \%$ and $54.4 \%$; and the number of eggs in $81.7 \%, 75.9 \%$ and $56.6 \%$, respectively.

Key words: Plant nematodes, alternative control, root-knot nematodes, natural compounds

\section{Introdução}

O controle de fitonematóides é uma tarefa difícil e o uso de produtos químicos tem sido restrito, pois além de caros, eles são altamente tóxicos ao meio ambiente e aos seres vivos. O uso de extratos vegetais com propriedades nematicidas no controle de fitonematóides representa mais uma alternativa para os pequenos produtores, com valor prático e econômico, e sem riscos de contaminação do ambiente. Várias pesquisas têm demonstrado o efeito nematicida dos extratos de diferentes plantas, quando aplicados diretamente ao solo (CHITWOOD, 2002). Com isso, o objetivo deste trabalho foi avaliar a eficiência da adição ao solo dos extratos aquosos provenientes de folhas de diversas espécies de plantas medicinais e outras utilizadas como adubos verdes sobre Meloidogyne javanica (Treub, 1885) Chitwood, 1949.

\section{Material e métodos}

O experimento foi conduzido na Universidade Federal de Viçosa, no Laboratório de Controle Biológico de Fitonematóides, localizado no núcleo de Biotecnologia Aplicada à Agropecuária (BIOAGRO) e em casa de vegetação do Departamento de Fitopatologia.

As espécies de plantas medicinais foram coletadas na coleção do "Grupo Entre Folhas", situado no Campus da Universidade Federal de Viçosa. As espécies de plantas usadas como adubos verdes foram obtidas através da semeadura em uma área experimental da Universidade Federal de Viçosa. As partes aéreas das plantas foram secas à sombra sobre bancadas em casa de vegetação, por aproximadamente cinco dias, variando o período de secagem conforme a espécie. As espécies estudadas foram divididas em dois grupos, e avaliadas em dois experimentos separados. No experimento 1, foram avaliadas as espécies do grupo 1: artemísia (Chrysanthemum parthenium (L.) Bernh.), bardana (Arctium lappa L.), capim-cidreira (Cymbopogon citratus (L.) Stapf), carqueja (Bacharis trimera (Less) D.C.), cavalinha (Equisetum sp.), erva-deSanta-Bárbara ou cinamomo (Melia azedarach L.), hortelã(Menthasp.), mamona(Ricinuscommunis L.), manjericão (Ocimum basilicum L.) e melão de São Caetano (Momordica charantia L). No experimento 2, foram avaliadas as espécies do grupo 2: arruda (Ruta graveolens L.), falso-boldo (Plectranthus barbatus Andrews), confrei (Symphitum officinalis L.), erva-de-bicho (Polygonum acre H.B.K.), feijão-de-porco (Canavalia ensiformis (L.) D.C.), funcho (Foeniculum vulgare Mill.), guiné (Petiveria alliacea L.), mentrasto (Ageratum conyzoides L.), mucuna-cinza (Mucuna pruriens var.pruriens (L.) D.C.) e nim (Azadirachta indica A. Juss.).

Os extratos aquosos das folhas foram obtidos seguindo o método descrito por Ferris e Zheng (1999), no qual se misturou $1 \mathrm{~g}$ de folhas secas de cada espécie de planta com $10 \mathrm{~mL}$ de água destilada, mantendo em repouso por 24 horas. Posteriormente, essa mistura foi filtrada em gaze e colocada em erlenmeyers cobertos com papel alumínio, identificados e utilizados logo em seguida.

Mudas de tomateiro (Lycopersicum esculentum Mill.) 'Santa Clara' foram transplantadas para vasos de plástico com capacidade de 2,0 L, contendo uma mistura de solo e areia na proporção de 1:1 (v:v), previamente esterilizado. Em seguida, o solo foi 
infestado com uma suspensão contendo 5.000 ovos de $M$. javanica. Os ovos foram extraídos utilizando a metodologia descrita Hussey e Barker (1973), modificado por Boneti e Ferraz (1981). No mesmo dia em que o solo foi infestado, $20 \mathrm{~mL}$ dos extratos vegetais foram adicionados ao solo de cada vaso, na forma de rega. Para o tratamento controle, foram aplicados $20 \mathrm{~mL}$ de água destilada ao solo. A aplicação foi realizada quinzenalmente por um período de 60 dias.

Aos 60 dias após a inoculação, foram avaliados o número de ovos e de galhas por sistema radicular, o peso fresco do sistema radicular e da parte aérea, e a altura de cada planta.

O delineamento estatístico utilizado foi $\mathrm{o}$ inteiramente casualizado, com 11 tratamentos e 7 repetições. Os dados obtidos foram submetidos à análise de variância e, quando necessário, ao teste de comparação de médias de Scott-Knot, ao nível de 5\% de probabilidade estatística (EUCLIDES, 1997). Os resultados obtidos da avaliação do número de galhas e de ovos/g de raiz, no experimento 1, foram transformados para $\sqrt{x}$. No experimento 2 , os dados da avaliação do número de ovos/g foram transformados para $\log _{10}(\mathrm{x})$.

\section{Resultados e discussão}

No experimento 1, a aplicação dos extratos de todas as plantas aumentou $(\mathrm{P} \leq 0,05)$ a altura e o peso da parte aérea das plantas, quando comparados com a testemunha (Tabela 1).

Tabela 1. Adição ao solo dos extratos aquosos de 10 espécies vegetais sobre a altura, peso da parte aérea, peso das raízes e número de galhas e de ovos por grama de raiz de tomateiro, aos 60 dias após a infestação do solo com 5.000 ovos de Meloidogyne javanica.

\begin{tabular}{|c|c|c|c|c|c|}
\hline Tratamentos & Altura (cm) & $\begin{array}{l}\text { Peso da parte } \\
\text { aérea }(\mathrm{g})\end{array}$ & $\begin{array}{l}\text { Peso das } \\
\text { raízes (g) }\end{array}$ & $\begin{array}{c}\mathbf{N}^{0} \text { de galhas / } \mathrm{g} \\
\text { de raiz }\end{array}$ & $\begin{array}{c}\mathrm{N}^{\mathbf{o}} \text { de ovos / g de } \\
\text { raiz }^{1}\end{array}$ \\
\hline Testemunha & $54,36 \mathrm{~b}$ & $18,40 \mathrm{~d}$ & $24,29 \mathrm{a}$ & $57,48 \mathrm{a}$ & $32.542,01 \mathrm{a}$ \\
\hline Manjericão & $\begin{array}{c}85,86 \mathrm{a} \\
(+57,90)^{*}\end{array}$ & $\begin{array}{c}42,79 \mathrm{a} \\
(+132,70)^{*}\end{array}$ & $\begin{array}{c}20,35 \mathrm{a} \\
(+88,68)^{*}\end{array}$ & $\begin{array}{r}31,25 \mathrm{~b} \\
(+45,60)^{*}\end{array}$ & $\begin{array}{c}11.029,11 \mathrm{c} \\
(+66,11)^{*}\end{array}$ \\
\hline Bardana & $\begin{array}{r}84,64 \mathrm{a} \\
(+55,70)\end{array}$ & $\begin{array}{c}45,01 \mathrm{a} \\
(+144,75)\end{array}$ & $\begin{array}{c}22,62 \mathrm{a} \\
(+109,83)\end{array}$ & $\begin{array}{l}19,69 \mathrm{c} \\
(+65,73)\end{array}$ & $\begin{array}{l}7.858,60 \mathrm{c} \\
(+75,85)\end{array}$ \\
\hline Cinamomo & $\begin{array}{r}83,21 \mathrm{a} \\
(+53,07)\end{array}$ & $\begin{array}{c}38,34 \mathrm{~b} \\
(+108,48)\end{array}$ & $\begin{array}{r}16,22 \mathrm{~b} \\
(+50,46)\end{array}$ & $\begin{array}{l}36,71 \mathrm{~b} \\
(+36,13)\end{array}$ & $\begin{array}{l}17.226,53 \mathrm{~b} \\
(+47,06)\end{array}$ \\
\hline Capim-cidreira & $\begin{array}{r}81,36 \mathrm{a} \\
(+49,66)\end{array}$ & $\begin{array}{c}36,31 \mathrm{~b} \\
(+97,44)\end{array}$ & $\begin{array}{r}18,48 \mathrm{a} \\
(+71,43)\end{array}$ & $\begin{array}{r}33,20 \mathrm{~b} \\
(+42,24)\end{array}$ & $\begin{array}{l}18.140,48 \mathrm{~b} \\
(+44,25)\end{array}$ \\
\hline Artemísia & $\begin{array}{r}81,14 \mathrm{a} \\
(+49,26)\end{array}$ & $\begin{array}{r}39,67 \mathrm{~b} \\
(+115,70)\end{array}$ & $\begin{array}{r}19,97 \mathrm{a} \\
(+85,25)\end{array}$ & $\begin{array}{r}31,47 \mathrm{~b} \\
(+45,25)\end{array}$ & $\begin{array}{l}17.406,93 \mathrm{~b} \\
(+46,51)\end{array}$ \\
\hline $\begin{array}{l}\text { Melão-de-São- } \\
\text { Caetano }\end{array}$ & $\begin{array}{r}78,79 \mathrm{a} \\
(+44,90)\end{array}$ & $\begin{array}{c}34,60 \mathrm{~b} \\
(+88,15)\end{array}$ & $\begin{array}{r}18,78 \mathrm{a} \\
(+74,21)\end{array}$ & $\begin{array}{l}32,80 \mathrm{~b} \\
(+43,00)\end{array}$ & $\begin{array}{l}16.823,93 \mathrm{~b} \\
(+48,30)\end{array}$ \\
\hline Hortelã & $\begin{array}{r}78,57 \mathrm{a} \\
(+44,54)\end{array}$ & $\begin{array}{c}36,67 \mathrm{~b} \\
(+99,40)\end{array}$ & $\begin{array}{r}16,51 \mathrm{~b} \\
(+53,15)\end{array}$ & $\begin{array}{l}13,99 \mathrm{c} \\
(+75,60)\end{array}$ & $\begin{array}{l}5.946,86 \mathrm{c} \\
(+81,72)\end{array}$ \\
\hline Cavalinha & $\begin{array}{r}78,43 \mathrm{a} \\
(+44,30)\end{array}$ & $\begin{array}{r}38,55 \mathrm{~b} \\
(+109,60)\end{array}$ & $\begin{array}{r}18,24 \mathrm{a} \\
(+69,20)\end{array}$ & $\begin{array}{r}38,52 \mathrm{~b} \\
(+32,99)\end{array}$ & $\begin{array}{l}22.239,20 \mathrm{~b} \\
(+31,66)\end{array}$ \\
\hline Carqueja & $\begin{array}{l}70,57 \mathrm{ab} \\
(+29,80)\end{array}$ & $\begin{array}{c}27,33 \mathrm{c} \\
(+48,61)\end{array}$ & $\begin{array}{r}15,64 \mathrm{~b} \\
(+45,08)\end{array}$ & $\begin{array}{r}37,73 \mathrm{~b} \\
(+34,35)\end{array}$ & $\begin{array}{l}17.655,48 \mathrm{~b} \\
(+45,74)\end{array}$ \\
\hline Mamona & $\begin{array}{r}88,36 \mathrm{a} \\
(+62,50)\end{array}$ & $\begin{array}{r}43,91 \mathrm{a} \\
(+138,77)\end{array}$ & $\begin{array}{r}21,42 \mathrm{a} \\
(+98,70)\end{array}$ & $\begin{array}{r}26,22 \mathrm{c} \\
(+54,40)\end{array}$ & $\begin{array}{l}14.108,51 \mathrm{c} \\
(+56,64)\end{array}$ \\
\hline $\mathrm{CV}$ & 12,70 & 13,26 & 16,42 & 21,04 & 23,06 \\
\hline
\end{tabular}

Média de 7 repetições. Médias seguidas pela mesma letra, nas colunas, não diferem entre si pelo teste Scott-Knot, a $5 \%$ de probabilidade. ${ }^{1}$ Valores transformados para a $\sqrt{x}$. *Percentual de redução (-) ou acréscimo (+) ao serem comparados os tratamentos com a testemunha só com nematóide. 
A adição ao solo do extrato aquoso de folhas de hortelã reduziu em 75,60\% o número de galhas por sistema radicular das plantas tratadas, seguido pelos extratos aquosos de folhas de bardana e de mamona, com redução em $65,73 \%$ e $54,40 \%$, respectivamente (Tabela 1). O número de ovos por sistema radicular foi reduzido pela aplicação ao solo dos extratos aquosos de folhas de hortelã, bardana e mamona na ordem de $81,72 \%, 75,85 \%$ e $56,64 \%$, respectivamente.

A atividade nematicida de extratos aquosos de várias espécies de hortelã sobre espécies de fitonematóides, como $M$. incognita (Kofoid; White) Chitwood, Ditylenchus myceliophagus Goodey, Xiphinema americanum Cobb, Longidorus sp., Hoplolaimus indicus Sher, Pratylenchus sp. e Helicotylenchus indicus Siddiqui, já foi demonstrada por vários pesquisadores (GANAI; KAUL; CHHABRA, 1992; HASEEB; KHAN; SAXENA, 1982; HASHIM; SEHAM; KHEIR, 1999; PANDEY; DWIVEDI, 2000; PANDEY et al., 2000). O óleo essencial de Mentha spicata L., M. piperata L., M. rotundifolia (L.) Huds e $M$. arvensis L. apresentam compostos com atividade nematicida comprovada, como o limonene, o piperitone e o menthol (OKA et al., 2000; PANDEY et al., 2000; SANGAWN et al., 1990). A hortelã, segundo Martins et al. (1995), possui compostos com ação vermífuga, que podem inativar ou mesmo matar vermes e lombrigas, talvez isso possa ser a razão da boa eficiência desta planta sobre os fitonematóides. Os resultados obtidos no presente ensaio e as características atribuídas a essa planta, indicam que ela deve ter atuado sobre a mobilidade e reprodução de $M$. javanica, visto que o número de galhas e de ovos foi reduzido em mais de $70 \%$. Sendo assim, a ação desta planta pode ter sido como nematostática, imobilizando o nematóide e assim diminuindo o número de galhas, e/ou apresentando um efeito nematicida, o qual diminuiria o número de nematóides e consequentemente o número de galhas e de ovos.

A atividade nematicida da bardana sobre $M$. javanica, neste ensaio, foi demonstrada com extratos aquosos adicionados ao solo. Dias et al. (2000), já haviam relatado a eficiência de extrato aquoso dessa planta, em testes 'in vitro', sobre M. incognita, apresentando atividade nematicida superior a 90\%. Esses resultados sugerem que esta planta possui compostos nematicidas que atuam sobre essas duas espécies, $M$. javanica e $M$. incognita, sendo uma planta indicada para estudos relacionados ao manejo de fitonematóides, visto que se tem pouca informação sobre ela.

Outra planta que apresentou pronunciada atividade nematicida no presente ensaio foi a mamona. Essa planta, segundo Rich, Rahi e Opperman (1989), já é conhecida por possuir certos compostos, como a ricina, que poderiam ser usados no controle de fitonematóides. Nandal e Bhatti (1986), ao adicionarem ao solo os extratos de folhas de mamona, observaram um efeito nematicida sobre M. javanica, reduzindo a eclosão de juvenis e a formação de galhas. Entretanto, os autores notaram que, ao final de 60 dias, o número de galhas e a eclosão de juvenis aumentaram; sugerindo que tal efeito poderia ser devido à degradação do componente tóxico. Dias et al. (2000) não observaram o efeito nematicida dos extratos aquosos de folhas de mamona sobre $M$. incognita, em testes 'in vitro'. Nesse caso, os autores sugeriram que o princípio ativo com atividade nematicida só possa ser liberado a partir da decomposição das folhas incorporadas ao solo. Outros autores avaliaram a incorporação ao solo de folhas desta planta e reportaram uma redução na população de diferentes espécies de fitonematóides (AKHTAR; ALAM, 1989; NANDAL; BHATTI, 1987). No presente ensaio, o extrato aquoso de mamona reduziu o número de galhas em $54,4 \%$ e de ovos em $56,6 \%$, diferindo estatisticamente da testemunha.

No experimento 2, a adição ao solo dos extratos foliares de todas as plantas, exceto mucuna-cinza, falso-boldo e erva-de-bicho, contribuíram para o aumento na altura das plantas tratadas, quando comparadas com a testemunha (Tabela 2). Os extratos de confrei e funcho aumentaram em $59,31 \%$ 
e $54,30 \%$ a altura das plantas e em $25,13 \%$ e $13,36 \%$ o peso da parte aérea. Em relação ao peso do sistema radicular, os extratos de feijão-de-porco, funcho, confrei, mucuna-cinza e guiné incrementaram o peso das raízes em 55,80\%, 43,33\%, 36,97\%, 34,96\% e $33,76 \%$, respectivamente.

Tabela 2. Adição ao solo dos extratos aquosos de 10 espécies vegetais sobre a altura, peso da parte aérea, peso das raízes e número de galhas e de ovos por grama de raiz de tomateiro, aos 60 dias após a infestação do solo com 5.000 ovos de Meloidogyne javanica.

\begin{tabular}{|c|c|c|c|c|}
\hline Tratamentos & Altura (cm) & $\begin{array}{l}\text { Peso da parte aérea } \\
\text { (g) }\end{array}$ & Peso das raízes (g) & $\underset{\text { raiz }}{\mathrm{N}^{0} \text { de galhas / g de }}$ \\
\hline Testemunha & $55,30 \mathrm{c}$ & $25,23 \mathrm{e}$ & $14,93 \mathrm{~b}$ & 24,76 \\
\hline Funcho & 88,08 a $(+59,31)^{*}$ & $56,78 \mathrm{a}(+25,13)^{*}$ & 21,40 a $(+43,33)^{*}$ & $15,45(+37,60)^{*}$ \\
\hline Confrei & 85,33 a $(+54,30)$ & 53,83 a $(+13,36)$ & $20,45 \mathrm{a}(+36,97)$ & $14,06(+43,21)$ \\
\hline Guiné & $77,92 \mathrm{~b}(+40,90)$ & $44,30 \mathrm{~b}(+75,60)$ & $19,97 \mathrm{a}(+33,76)$ & $13,16(+46,85)$ \\
\hline Feijão-de-porco & $73,58 \mathrm{~b}(+33,10)$ & $46,08 \mathrm{~b}(+82,72)$ & 23,26 a $(+55,80)$ & $15,37(+37,96)$ \\
\hline $\mathrm{Nim}$ & $72,70 \mathrm{~b}(+31,46)$ & $39,08 \mathrm{c}(+54,97)$ & $17,09 \mathrm{~b}(+14,53)$ & $16,57(+33,10)$ \\
\hline Mentrasto & $72,58 \mathrm{~b}(+31,30)$ & $41,63 \mathrm{~b}(+65,00)$ & $18,86 \mathrm{~b}(+26,32)$ & $16,39(+33,80)$ \\
\hline Arruda & $72,42 \mathrm{~b}(+30,96)$ & $44,16 \mathrm{~b}(+75,03)$ & $17,71 \mathrm{~b}(+18,62)$ & $14,52(+41,35)$ \\
\hline Mucuna-cinza & 63,42 c $(+14,70)$ & $34,40 \mathrm{c}(+36,34)$ & 20,15 a $(+34,96)$ & $19,64(+20,70)$ \\
\hline Falso-boldo & $62,58 \mathrm{c}(+13,20)$ & $33,70 \mathrm{c}(+33,60)$ & $17,55 \mathrm{~b}(+17,55)$ & $15,52(+37,31)$ \\
\hline Erva-de-bicho & $57,00 \mathrm{c} \quad(+3,10)$ & $30,91 \mathrm{~d}(+22,10)$ & $17,87 \mathrm{~b}(+19,69)$ & $16,48(+33,44)$ \\
\hline $\mathbf{C V}$ & 12,85 & 10,93 & 16,95 & 40,57 \\
\hline
\end{tabular}

Média de 7 repetições. Médias seguidas pela mesma letra, nas colunas, não diferem entre si pelo teste Scott-Knot, a $5 \%$ de probabilidade.

A adição ao solo dos extratos aquosos das plantas avaliadas no experimento 2 não reduziu o número de galhas e de ovos por sistema radicular.

Embora no presente experimento nenhuma planta avaliada tenha diferido estatisticamente da testemunha, quanto ao número de galhas e de ovos de M. javanica, dentre as espécies de plantas estudadas, algumas já demonstraram efeito sobrefitonematóides (CHITWOOD, 2002). Provavelmente o princípio ativo tenha sido degradado devido ao modo de extração, pois há compostos que são altamente solúveis em água, sendo necessário outro tipo de solvente para que se possa extraí-los e mantê-los ativos por mais tempo.

De acordo com os resultados obtidos nestes experimentos, conclui-se que os extratos de hortelã, bardana e mamona, adicionados ao solo, apresentaram eficiência em controlar $M$. javanica. $\mathrm{O}$ uso de extratos vegetais brutos no manejo de fitonematóides em relação aos produtos sintéticos ou purificados pode resultar em benefícios econômicos, em casos onde não seja necessária a aplicação de grandes volumes do extrato, e este apresente uma boa eficiência em reduzir a população de fitonematóides (CHITWOOD, 2002).

\section{Agradecimentos}

Ao Grupo Entre Folhas, situado no Campus da Universidade Federal de Viçosa, ao Instituto Agronômico do Paraná e a Sementes Piraí, por cederem sementes e espécies vegetais avaliadas e ao CNPq pelo apoio financeiro. 


\section{Referências}

AKHTAR, M.; ALAM, M.M. Evaluation of nematicidal potential in some medicinal plants. International Nematology Network Newsletter, Haleigh, v. 6, n. 1, p. 8-10, 1989.

BONETI, J. I. S.; FERRAZ, S. Modificação do método para extração de ovos para Meloidogyne exigua em raízes de cafeeiro. Fitopatologia Brasileira, Brasília, v. 6, p. 553, 1981. Resumo.

CHITWOOD, D. J. Phytochemical based strategies for nematode control. Annual Review of Phytopathology, Palo Alto, v. 40, p. 221-249, 2002.

DIAS, C. R.; SCHWAN, A.V.; EZEQUIEL, D. P.; SARMENTO, M. C.; FERRAZ, S. Efeito de extratos aquosos de plantas medicinais na sobrevivência de juvenis de Meloidogyne incognita. Nematologia brasileira, Campinas, v. 24, n. 2, p. 203-210, 2000.

EUCLIDES, R. F. SAEG - Sistema para Análises Estatísticas e Genéticas. Versão 7.1. Viçosa: Universidade Federal de Viçosa, 1997. 150 p. (Manual do Usuário).

FERRIS, H.; ZHENG, L. Plant sources of chinese herbal remedies: Effects on Pratylenchus vulnus and Meloidogyne javanica. Journal of Nematology, College Park, v. 31, n. 3, p. 241-263, 1999.

GANAI, G. H.; KAUL, V. K.; CHHABRA, H. K. Nematicidal action of leaf extracts of neem, Mentha and Parthenium against Ditylenchus myceliophagus. Plant Disease Research, Ludhiana, v. 7, n. 2, p. 279-281, 1992.

HASEEB, A.; KHAN, A. M.; SAXENA, S. K. Toxicity of leaf extracts of plants to root-knot and reniform nematodes. Indian Journal of Parasitology, Lucknow, v. 6, n. 1, p. 119-120, 1982.

HASHIM, E. F.; SEHAM, K. A. A.; KHEIR, A. A. Nematicidal activity of some labiataceous plant extracts on Meloidogyne incognita. Annals of Agricultural Science, Cairo, v. 44, n. 1, p. 447-457, 1999.
HUSSEY, R. S.; BARKER, K. R. A comparison of methods of collecting inocula of Meloidogyne spp., incluinding a new technique. Plant Disease Reporter, St. Paul, 57:1025-1028. 1973.

MARTINS, E. R.; CASTRO, D. M.; CASTELLANI, D. C.; DIAS, J. E. Plantas medicinais. Viçosa: Imprensa Universitária, 1995.

NANDAL, S. N.; BHATTI, D. S. Effect of some weed and shrub extracts on penetration and gall formation by Meloidogyne javanica on brinjal. Nematologia Mediterranea, Bari, v. 15, n. 1/2, p. 159-162, 1987.

. Influence of four plant extracts on the hatching of Meloidogyne javanica and invasion of host roots. Nematologia Mediterranea, Bari, v. 14, n. 1/2, p. 291294, 1986.

OKA, Y.; NACAR, S.; PUTIEVSKY, E.; RAVID, U.; YANIV, Z.; SPIEGEL, Y. Nematicidal activity of essential oils and their components against the root-knot nematode. Nematology, Leiden, v. 90, n. 7, p.710-715, 2000 .

PANDEY, R.; DWIVEDI, B. K. Comparative study of different plant extracts for their nematicidal potential. Current Nematology, Allahabad, v. 11, n. 1/2, p. 39-43, 2000.

PANDEY, R.; KALRA, A.; TANDON, S.; MEHROTRA, N.; SINGH, H. N.; KUMAR, S. Essential oils as potent sources of nematicidal compounds. Journal of Phytopathology, Berlin, v. 148, n. 7/8, p. 501-502, 2000.

RICH, J. R.; RAHI, G. S.; OPPERMAN, C. H. Influence of the castor bean (Ricinus communis) lectin (Ricin) on motility of Meloidogyne incognita. Nematropica, Bradenton, v. 19, n. 1, p. 99-103, 1989.

SANGAWN, N. K.; VERMA, B. S.; VERMA, K. K.; DHINDSA, K. S. Nematicidal activity of some essential plant oils. Pesticide Science, Oxford, v. 28, n. 3, p. 331335, 1990. 